IRA-International Journal of Applied Sciences ISSN 2455-4499; Vol.05, Issue 01 (2016)

Institute of Research Advances

Pg. no. 22-28

http://research-advances.org/index.php/IRAJAS

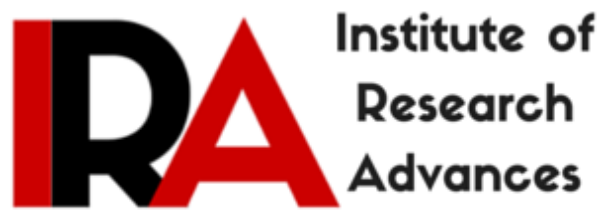

\title{
Association of Pregnancy Anxiety and the Risk of Hypertensive Disorder during Pregnancy
}

\section{Sharda Ghoghre}

Assistant Prof., Department of Psychology,

Govt. Kirodimal Arts \& Science College, Raigarh, Chattisgarh, India.

Type of Review: Peer Reviewed.

DOI: http://dx.doi.org/10.21013/jas.v5.n1.p4

\section{How to cite this paper:}

Ghoghre, S. (2016). Association of Pregnancy Anxiety and the Risk of Hypertensive Disorder during Pregnancy. IRA-International Journal of Applied Sciences (ISSN 24554499), 5(1), 22-28. doi:http://dx.doi.org/10.21013/jas.v5.n1.p4

(C) Institute of Research Advances

\section{(cc) EY-NO}

This work is licensed under a Creative Commons Attribution-Non Commercial 4.0 International License subject to proper citation to the publication source of the work.

Disclaimer: The scholarly papers as reviewed and published by the Institute of Research Advances (IRA) are the views and opinions of their respective authors and are not the views or opinions of the IRA. The IRA disclaims of any harm or loss caused due to the published content to any party. 


\begin{abstract}
Present research study examines the association of pregnancy anxiety and the risk of hypertensive disorder during pregnancy. It is a cross sectional study spread over a period of one and half year. This research is conducted on 200 pregnant women from different maternity homes of Betul \& Bhopal District from Madhya Pradesh, India. Levin Pregnancy anxiety scale and SCL -90R (Dergotis,1994) was used to assess the level of pregnancy anxiety among pregnant women having hypertensive disorder or without hypertensive disorder. One way analysis of variance was used for analysis of data to see a significant relationship between pregnancy anxiety and risk of hypertensive disorder among patient group and normal pregnant women's. The results of the study revealed a significant relationship of pregnancy anxiety among pregnant women having hypertensive disorder in comparison to normal pregnant women's.
\end{abstract}

KEYWORDS: Hypertensive disorder, pregnancy anxiety, preeclampsia, gestational and chronic hypertension

\title{
INTRODUCTION
}

Hypertension is the most common medical problem encountered during pregnancy, complicating 2-3\% of pregnancies. Hypertensive disorders during pregnancy are classified into 4 categories, as recommended by the National High Blood Pressure Program Working Group on High Blood Pressure in Pregnancy: 1) chronic hypertension, 2) preeclampsia-eclampsia, 3) preeclampsia superimposed on chronic hypertension identified in the latter half of pregnancy (Report of the National High Blood Pressure Education Program Working Group on High Blood Pressure in Pregnancy, 2000).

Psychological factors may also have an important role to play in the development of anxiety and depression disorders at this time. Often the expectant mother has concerns over the health of the child, the change in lifestyle likely to occur in her own life after the birth of the child, her own ability to be a good mother, and finances. There are also instances where the pregnancy is unexpected or unwanted, which may further increase stress and anxiety. For some women, pregnancy may bring to mind painful events in their lives with their own parents. Psychologically healthy woman often find a pregnancy a means of selfrealization. Other women use pregnancy to diminish self-doubts about feminity or to reassure that they can function as women in the most basic sense. Still others view pregnancy negatively; they may fear childbirth or feel inadequate about mothering. At least one in ten mothers in all levels of society, and regardless of socioeconomic conditions experience clinical depression and/or anxiety before and up to a year after childbirth. The trimesters can bring their characteristic challenges and rewards, the nausea, fatigue and emotionality. For many women, the first and their trimesters are difficult (Saddock, 2003).

\section{CURRENT CONCEPTS}

Pregnancy is a time of growth and hopes (Hamid et al., 2008; Schroeder, 1996) and perceived by many pregnant mothers as a period of happiness in anticipation of motherhood. Women hope for a smooth journey in pregnancy without any complications and a normal fetal development. However, not all pregnant mothers experience joy and cheerfulness as some may experience a high anxiety and depression. Pregnancy is a time of great emotional change for women, often producing increased stress and anxiety. Gonadal steroid levels have been reported with as much as a 100 -fold variation in serum estrogen levels and a 1000-fold change in serum progesterone levels during pregnancy. These changes can exacerbate such emotional difficulties resulting in anxiety, depression, and other psychiatric disorders.

Cattle \& Scheier (1958) described anxiety as an unpleasant emotional experience characterized by a feeling of impending danger. The consequences of anxiety are many and include an inability to communicate with love ones. Similarly, Light \& Fenster (1974) defined anxiety as an uneasiness of mind resulting from an emotionally stressful situation. The transition to motherhood is a stress producing 
process involving adaptation under the most favorable circumstances (Mandeville \& Troiano, 1999). In the prenatal stage, women have fantasies. These involve the creativity of characteristics of the mental image of their unborn children, derived from the affiliation. The most frequently reported fantasies with negative content related to fetal abnormalities, violence and defective maternal care giving (Sorenson \& Schuelke, 1999). However, it has been well established that all women experiences are increase in anxiety during their pregnancies. Although the degrees of anxiety are varied depending upon the emotional makes up of the women the specific meaning that the pregnancy has for her with its concomitant external factors.

Research study show anxiety in women was related to many factors including image, self health, well being of the fetus, labor and delivery or relationship with spouses, and involves the family economic status as well. Some forms of anxiety are always present during pregnancy. Anxiety arises from unmet expectations and acquired needs relating to prestige, status, esteem, and consideration of oneself which might not be met by pregnancy and expected motherhood (Clark, et al., 1979). Various descriptive and exploratory studies show that there are certain factors when pregnant women may experience specific anxiety and intense fears, such as fear of incompetence and concerns about pain and loss of control during delivery, fear for their own life and the life of their baby, and worries about changes in their personal life due to pregnancy and childbirth (Dunkel \& Schetter, 1998; Sjögren, 1997).

Several studies conducted in recent years which demonstrated that prenatal symptoms of anxiety and depression are related to a range of obstetric complications including recurrent spontaneous abortion (Sugiura-Ogasawara, Aoki, \& Kitamura, 2002) and spontaneous preterm labor (Dayan, Savoye, \& Thoulin, 2002), which often results in preterm delivery. Moreover, prenatal anxiety and depression at 32 weeks gestation, but not in early pregnancy, has been associated with an increased risk of neonatal intensive care unit admissions, use of epidural analgesia, and operative deliveries, although the effect of depression on operative deliveries was non-significant when controlling for analgesia administration (Chung, Chiu, \& Lee, 2001). Both anxiety and depression have been linked to increased risk of preeclampsia and other high-risk conditions (Kurki, Mattila, \& Ylikorkala, 2000). Teixeira, Fisk, \& Glover (1999) found an association between elevated scores on the State Trait Anxiety Inventory (STAI; Spielberger, 1983) and increased uterine artery resistance, which ultimately results in decreased blood flow to the fetus and has been suggested as a potential mediating mechanism in the association between maternal emotional state during pregnancy and neonatal outcome.

The emotional stress or anxiety in the mother during pregnancy may be associated with difficulty of labor and delivery and adverse effects upon the fetus and neonate (Light \& Fanster, 1974). Many researchers have reported the dimensions of prenatal anxiety and their influence on pregnancy outcomes such as premature labor, birth complications, low birth weight, and including long term neuro developmental effects on the infant (Glover, 1977; Paarberg, et al.1999). Significantly, pregnant women with high anxiety have low attachment to their babies, and also assume ineffective maternal role adaptation (Avant, 1981; Mercer et. al., 1988).

Theut and others (1988) conducted the first empirical study in which data demonstrated the grief responses in a subsequent pregnancy. In that study, 25 expectant couples who had experienced perinatal loss were compared with 31 first time expectant couples. The results indicated that women with perinatal loss had more specific anxiety during pregnancy than the other group. Hunfeld \& others (1996) conducted a study to determine the quality of life and anxiety in pregnancy during 18-21 weeks gestation after late pregnancy loss (16 weeks) comparing between 24 pregnant women with, and 26 women without a pevious perinatal loss. The result showed that women who have experienced perinatal loss had a lower quality of life and greater anxiety related pregnancy. This finding indicated a result similar to that of Theut and others (1988).

Additionally, Statham and Green (1994) found that pregnant women with a history of miscarriage 
and no living children were associated with a higher and more enduring level of anxiety. Hughes and others (1999) conducted the cohort study to assess womens symptoms of depression and anxiety during pregnancy and the postpartum years in the pregnancy after stillbirth. The subjects were 60 pregnant women who had previous stillbirth after 18 weeks gestation. The control group consisted of 60 pregnant women without history of perinatal loss were significantly more depressed and had greater anxiety than the control group.

In addition, many factors may influence anxiety in pregnant women involving themselves, unborn infants, significant persons, and economic status during pregnancy. With regards to pregnant women who experienced prenatal loss felt more anxiety than pregnant women without such a history. Health care providers should concern and pay more attention in this issue because anxiety can affect both of pregnant women and her fetus including delayed or decreased prenatal attachment with the child in the current pregnancy (Armstron \& Hutti, 1998; Rillstone \& Hutchison, 2001).

A few research studies have shown pregnancy is considered a period of well being and happiness, and was once thought to protect women from anxiety and depression (Buist A, et al., 2000). Women for childbearing age frequently suffer from major depression and anxiety (D' Alfonso A et al., 2002; Gaynes BN et al., 2005). A large number of studies have reported that depression and anxiety are more prevalent during pregnancy in all over the world. But few study reported that depression was common after delivery. The striking feature of anxiety is that it impacts both mother and infant. Its effects include cognitive, emotional and social difficulties, difficulty in attachment and infant development. The result of the study show that level of anxiety during pregnancy is significantly related to Hypertensive disorders i.e preeclampsia this finding is similar to the previous data and findings of Kurki et al., 2000 studies demonstrate the link between psychological factors and preeclampsia, who reported higher levels of anxiety or depressive symptoms early in pregnancy, prior to the development of preeclampsia, were 2-3 times more likely to develop the disorder than their less anxious or depressed counterparts (Kurki et al., 2000; Piyasil, 1998; Raskin et al., 1990). The relationship between antenatal and postnatal symptom of depression has been investigated by Josefsson et al (2002) who showed that $6 \%$ of the women had symptoms both during pregnancy and postpartum. Another study that is not consistent with the findings of the study, by Vollebregt et al (2008) investigated the association of preeclampsia and gestational hypertension with psychosocial stress in the first half of pregnancy and found that anxiety, pregnancy related anxiety or depression had no effect on the incidence of preeclampsia and gestational hypertension. While not found in all studies (Nisell, Larsson, \& Wager, 1989), evidence also supports a link between self-reported stressful life events and likelihood of preeclampsia (Hetzel, Bruer, \& Poidevin, 1961). Gestational hypertension and subsequent preeclampsia has also been linked to untreated depression during pregnancy. Psychopathology during pregnancy is thought to affect the uterine environment and, therefore, could have an effect on fetal outcome. Current theories suggest that the symptom of anxiety and depression increases excretion of vasoactive hormones in the mother, and these hormones then mediate birth outcome. More research is needed to find out the exact mechanism which is responsible for preeclampsia.

In conclusion, several studies show the role of psychological variables in maternal and fetal complication during pregnancy and their consequences. For elimination of these effect proper psychological intervention must be used.

\section{OBJECTIVE:}

1. To compare the samples of pregnant women with hypertensive disorder and normal pregnant women on the severity of anxiety.

2. To examine the relationships of anxiety with the risk of hypertensive disorder among pregnant women. 


\section{HYPOTHESIS:}

1. Pregnant women with hypertensive disorder will show significantly higher levels of anxiety than normal pregnant women without preeclampsia

2. Presence of anxiety symptom during pregnancy would be significantly related to the risk and severity of hypertensive disorders.

\section{METHODOLOGY \\ Sample}

A cross sectional study was conducted on $200(\mathrm{n}=200)$ pregnant women. The study was conducted in the obstetric wards of hospital of Madhya Pradesh. Mainly two districts; were taken in the study; Bhopal and Betul. The sample includes one hundred cases of pregnant women with hypertensive disorders. Hypertensive disorders cases was identified by daily monitoring of all new admission to ante partum wards, emergency wards and labor room and delivery wards of the hospitals. Control group consisted women without hypertensive disorder, uncomplicated by pregnancy induced hypertension or proteinuria. It comprises of 100 pregnant women without hypertensive disorder matched on age, educational level, marital status, family type, income and other relevant variables. This was a cross sectional study and the sampling technique was of convenience sampling. The study was spread over a period of more than one year (2007-09).

\section{Design}

In order to examine the relationships of different measure, a cross sectional design was used. One way analysis of variance was used to see the significant relationship between groups on the measure of pregnancy anxiety.

\section{Tool}

Pregnancy anxiety scale is developed by Levin in 1991.It is a 10 item scale showing maternal anxiety during pregnancy. It is intended to be useful as a diagnostic tool related to the pregnancy experience and more useful than general anxiety inventories. The 10 items are scored $0=$ no, $1=y e s$, and summed.

The 13 item PAS was tested on 266 postpartum mothers, largely Africa-America and Hispanic, and show reliability of .63. Factor analysis shows three factors: anxiety about being pregnant (items 1 to 3), anxiety about childbirth (items 4 to 7), and anxiety about hospitalization (items 8 to 10). Based on these measurement characteristics three items were removed, leaving 10.

To measure anxiety symptoms, only anxiety part of the SCL-90-R was used. It is developed by Dergotis (1994). It is a 90-item self-report measure of multidimensional mental health symptoms, because: (a) it requires only elementary school reading skills due to its brief, simply worded items; (b) it assesses patients' current symptoms within a specified and optimal point-in-time (i.e., the past 7 days) that may better evaluate mental health changes; and (c) it has often been used in research.

Patients are instructed to rate mental health symptoms that have "bothered" them within the past 7 days on a 5-point scale (0-4) ranging from "Not at All" (i.e., a "0" rating) to "Extremely" (i.e., a "4" rating). The SCL-90-R includes three global index scales and nine symptom scales that were based on factor analysis and that include diagnostic-specific and non-specific symptoms. The symptom scales include the Somatization, Obsessive-Compulsive, Interpersonal Sensitivity, Depression, Anxiety, Hostility, Phobic Anxiety, Paranoid Ideation, and Psychoticism scales. The SCL-90-R index scales include the Positive Symptom Total, Positive Symptom Distress Index, and Global Severity Index.

\section{Procedure}

Different maternity homes and hospital of Betul and Bhopal were listed. Then the permission was taken from the higher authority to conduct this research. After that participants having hypertensive disorder or without hypertensive disorder were selected, then consent was taken from them. In a quiet place (Doctors duty room) sitting arrangement was done, after that good rapport was established with the 
participants, necessary instructions and guidelines were provided to them for properly responding the questionnaire. The purpose of the study was explained and participants were assured that their responses will be kept confidential and will be used for research and academic purpose only. After this, question were asked to them and requested to give answer as per the instructions provided to them. After that we thanks to participants for heartily cooperation to complete their questionnaire. All other necessary information regarding participants was also collected from hospital staff and hospital record book.

\section{RESULTS AND DISCUSSION}

The main motive of the study to see the association between pregnancy anxiety and risk and severity of hypertensive disorders during pregnancy. The early detection of symptoms can facilitate the timely treatment and prevent the deterioration of the condition. Results of one way ANOVA revealed that women with hypertensive disorder significantly differed from normal pregnant women on the measures of anxiety

\section{Table 1}

Mean scores, SD and F Value to show the level of anxiety in groups of patient with hypertensive disorder and normal pregnant women.

\begin{tabular}{lllll}
\hline Variables & $\boldsymbol{N}$ & $\boldsymbol{M}$ & $\boldsymbol{S D}$ & $\boldsymbol{F}(\mathbf{1}, \mathbf{1 9 8})$ \\
\hline Anxiety & & & & \\
Patient & 100 & 1.40 & 0.57 & $19.89^{* *}$ \\
Control & 100 & 1.11 & 0.34 & \\
\hline
\end{tabular}

$* * P<.01$.

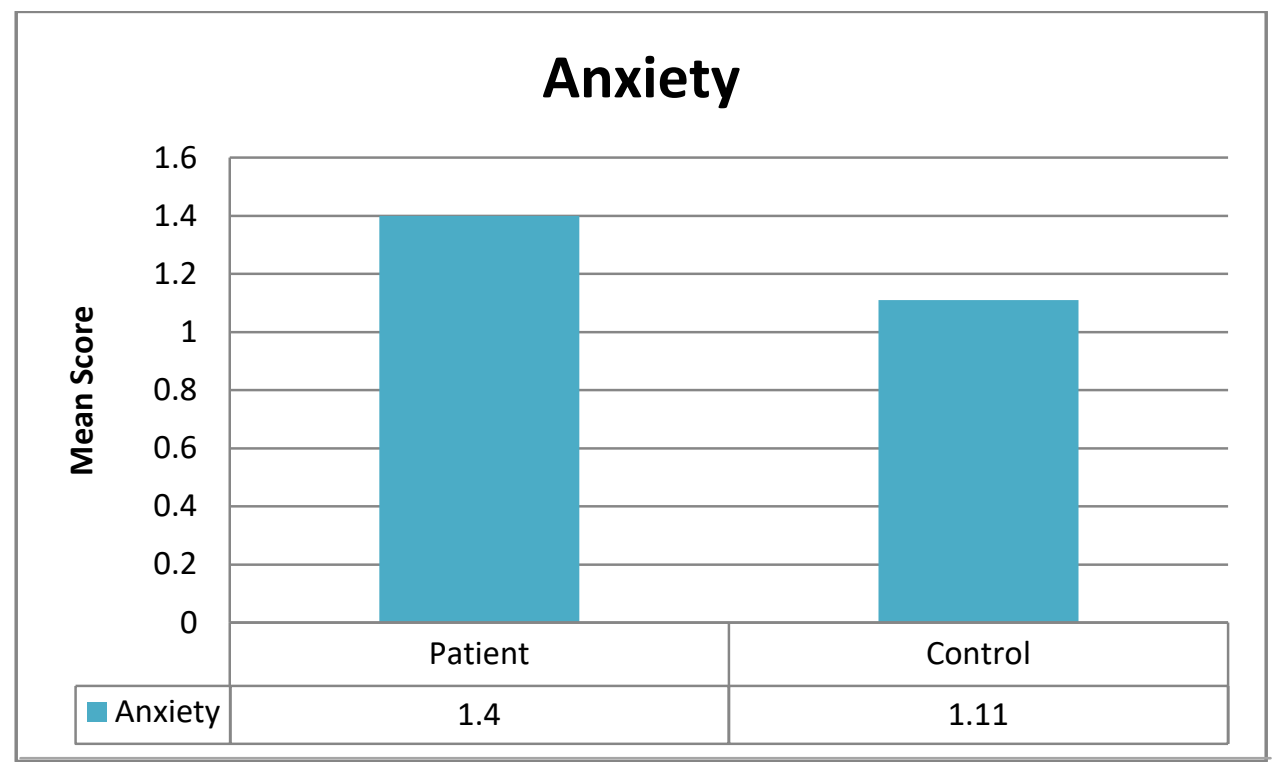

Figure 1. Mean score of groups on the level of anxiety

To examine the relationship between groups on the level of anxiety, one way ANOVA was used. A summary of between group analysis of variance for the measures of anxiety shown in table 1, that revealed a significant relationship between groups and level of anxiety, $F(1,198)=19.89, p<.01$. Mean scores indicate that women with Hypertensive disorders had higher level of anxiety $(M=1.40, S D=0.57)$ 
in comparison to the control group $(M=1.11, S D=0.34)$.

\section{CONCLUSION}

Finally we concluded that the presence of anxiety symptom during pregnancy is associated with risk factors of hypertensive disorder during pregnancy. So it's important for family member to provide emotional and psychological support to overcome from stress and anxiety during pregnancy.

\section{REFERENCE}

1. American College of Obstetricians and Gynecologists Committee on Obstetric Practice. (2002). Diagnosis and management of preeclampsia and eclampsia. International Journal of Gynecology and Obstetrics, 77, 67-75.

2. Buist, A. (2000), Managing depression in pregnancy. Australian Family Physician 29, 663-667.

3. Buist, A. E., Milgrom, J., Morse, C., Durkin, S. and Rolls, C. (2000). Integrating services in the recognition and management of postpartum depression. Australian Journal of Primary Health Interchange, 6(3-4), 74-79.

4. D'Alfonso, A., Iovenitti, P., Casacchia, M., Carta, G. (2002). Disturbances of humour in postpartum: our experience. Clinical and Experimental Obstetrics \& Gynecology ,29, 207-211.

5. Dayan. J, Crevcuil. C, Herlicoveiz. M, Herbel. C, Baranger. E, Savoye C, Thouin. A, (2002). Role of anxiety and depression in the onset of spontaneous preterm labour. American Journal of Epidemiology. 155(4):293-301.

6. Dunkel-Schetter, C. (1998). Maternal stress and preterm delivery. Prenatal and Neonatal medicines, 3, 39-42.

7. Hamid, F., Asif, A., \& Haider, I.I. (2008). Study of anxiety and depression during pregnancy. Pakistan Journal of Medical Sciences, 24 (6), 861-864.

8. Light, H.K., \& Fenster, C.(1974). Maternal concern during pregnancy. American Journal of Obstetrics and Gynecology, 118,45-6.

9. Kurki, T., Hiilesmaa, V., Raitasalo, R., Mattila, H., \& Ylikorkala, O. (2000). Depression and anxiety in early pregnancy and risk for preeclampsia. Obstetric and Gynecology, 95, 487490.

10. Kurki, T., Hiilesmaa, V., Raitasalo, R., Mattila, H., \& Ylikorkala, O. (2000). Depression and anxiety in early pregnancy and risk for preeclampsia. Obstetric and Gynecology, 95, 487-490.

11. Mandeville, L. \& Troiano, N. (1999). High risk \& critical care intrapartum nursing $\left(2^{\text {nd }}\right.$ ed.) Philadelphia: Liipponcort.

12. National High Blood Pressure Education Program (2000). Working Group Report on High Blood Pressure in Pregnancy. Md: National institutes of health; NIH publication No. 00- 3029.

13. Saddock K. (2003) Synopsis of Psychiatry. Behavioral Sciences and Clinical Psychiatry. 9th edition. Lippincott Williams \& Wilkins USA.

14. Sorenson, D.S., \& Schuelke, P.(1999). Fantasies of the unborn among pregnant women. Maternal-Child Nursing Journal, 24, 92-97.

15. Sugiura-Ogasawara, M., Furukawa, T.A., Nakano, Y., Hori, S., Aoki, K., \& Kitamura, T. (2002). Depression as a potential causal factor in subsequent miscarriage in recurrent spontaneous aborters. Human Reproduction, 17(10), 2580-2584.

16. Texeira, J.M.A., Fisk, N.M., \& Glover, V. (1999). Association between maternal anxiety in pregnancy and increased uterine artery resistance index: cohort based study. British Medical Journal, 318,153-157. 